\title{
The Effect of Melatonin on the Functional State of the Hemostatic System and Osmoregulatory Kidney Function in Rats With Chronic Molybdenum Intoxication
}

\author{
Brin V.B. ${ }^{1,2, *}$ Gagloeva E.M. ${ }^{3}$ Moldovan T.V. ${ }^{3}$ Botsieva N.V. ${ }^{3}$ \\ ${ }^{1}$ Department of Normal Physiology FSBEI HE SOGMA Ministry of Health of the Russian Federation, North Ossetia- \\ Alania, Vladikavkaz, Russia \\ ${ }^{2}$ Department of Physiology and Pathology of Visceral Systems, IBMI VSC RAS, North Ossetia-Alania, Vladikavkaz, \\ Russia \\ ${ }^{3}$ Department of Normal Physiology, Federal State Budgetary Educational Institution of Higher Education in the \\ Ministry of Health of the Russian Federation, North Ossetia-Alania, Vladikavkaz, Russia \\ *Corresponding author. Email: vbbrin@yandex.ru
}

\begin{abstract}
This work aimed to study the relationship between the mechanisms of the formation of toxic coagulopathy and nephrotoxic effects of molybdenum, as well as the development of a pathogenetically substantiated method for the prevention of chronic toxic coagulopathy and nephropathy using the pineal gland hormone melatonin. A solution of ammonium paramolybdate was administered to Wistar rats for two months at a dose of $50 \mathrm{mg} / \mathrm{kg}$ through a tube into the stomach. Against the background of chronic molybdenum intoxication, a solution of melatonin was administered intragastrically at a dose of $5 \mathrm{mg} / \mathrm{kg}$ of body weight. Under the conditions of a 6-hour spontaneous diuresis, a study was conducted of the main processes of urination, indicators of the cortico-papillary gradient of the concentration of urea, and sodium in the layers of kidney tissue. We studied the state of the hemostatic system and lipid peroxidation processes after two months of experiments. The experiments showed that the prophylactic administration of melatonin causes a decrease in the severity of pathological changes in the indicators of vascular-platelet and coagulation hemostasis, anticoagulant, fibrinolytic link, a decrease in the level of thrombinemia associated with the restoration of indicators of the osmoregulatory function of the kidneys. Positive effects correlate with the restoration of the level of lipid peroxidation products and the activity of antioxidant blood enzymes. The results of the study allow reasonably recommending the use of the antioxidant melatonin to develop methods for correcting impaired renal function when molybdenum compounds are exposed to the body.
\end{abstract}

Keywords: melatonin, molybdenum, renal function, hemostasis.

\section{INTRODUCTION}

Molybdenum is an essential trace element necessary for the regular implementation of vital processes. It plays a vital role in tissue respiration and is an integral part of enzymes such as aldehyde oxidase, xanthine oxidase, and sulfite oxidase. These enzymes are responsible for the processes of DNA and RNA metabolism, affect protein synthesis, carbohydrate metabolism, the autonomic nervous system, endocrine function, and other processes [1]. At the same time, the prolonged intake of molybdenum into the body as a result of anthropogenic environmental pollution can lead to excessive accumulation of heavy metal in human organs and tissues and multiple organ-functional structural disorders [7, 12]. At the same time, the kidneys are exposed to a high toxic load, since they are the primary system for removing xenobiotics from the body [7,9]. It was shown that molybdenum intoxication is accompanied by activation of lipid peroxidation, the formation of oxidative stress and damage to membrane structures $[1,4]$.

Numerous experimental and clinical studies suggest that for any pathological processes in the body, the functional blood coagulation system is one of the first to react, changing its functioning parameters $[6,8,10]$. Changes in the hemostatic system accompany the pathology of the cardiovascular system, hypertension, cerebrovascular accident, diabetes mellitus, liver, and kidney pathology [8]. Hematological indicators in heavy industry workers depend on the length of service [1]. However, the state of the coagulation system during molybdenum intoxication remains poorly understood. The problem of creating and developing effective methods of 
pathogenetic prophylaxis and treatment of chronic intoxication with molybdenum and its compounds is relevant $[1,7]$.

As a means of preventing molybdenum intoxication, we used a synthetic analog of the hormone of the pineal gland melatonin Melaxen [2,3]. Previously, melatonin was used for preventing the pathology of the cardiovascular system, liver, and kidneys in an experiment in rats with chronic toxic effects of lead, zinc, copper, and cobalt $[4,5,13]$. Data on the possibility of using melatonin for the prevention of the toxic effects of molybdenum were not found in the available literature.

This work aimed to study the relationship between the mechanisms of formation of toxic coagulopathy and the nephrotoxic effects of molybdenum, the development of a pathogenetically substantiated method for the prevention of chronic toxic coagulopathy and nephropathy using a natural antioxidant - the pineal gland hormone melatonin.

\section{METHODS AND MATERIALS}

The experiments were performed on 40 male Wistar rats weighing $200-250$ g. Group 1 (10 rats) were intact animals. Group 2 (10 rats) - animals with intragastric administration of melatonin at a dose of $5 \mathrm{mg} / \mathrm{kg}$ for two months. Group 3 (10 rats) - animals with intragastric administration of a solution of ammonium paramolybdate at a dose of $50 \mathrm{mg} / \mathrm{kg}$ every day for two months [4]. Group 4 (10 rats) - animals with chronic molybdenum intoxication in combination with intragastric administration of melatonin for two months. Experimental animals during the study period were kept in standard vivarium conditions with free access to water and food.

The study of the state of the urinary function of the kidneys was carried out by measuring the volume of a 6hour spontaneous diuresis. The concentration of creatinine and total protein in urine and blood plasma was determined on a SOLAR 300 spectrophotometer using Agat-Med kits (Moscow, Russia). The osmolarity of urine and blood plasma was determined by cryoscopy on an OSMOMAT-2 millionometer. The extraction of sodium from the tissues of the kidney was performed $0.75 \mathrm{~N}$ nitric acid. The sodium concentration was determined using a flame automatic photometer "FPA-2." The urea content in the layers of kidney tissue was determined by the method of S.G. Hasanova (1962) in our modification (rational proposal No. 241, dated December 20, 2006; rational proposal No. 242, dated December 20, 2006). The clearance of endogenous creatinine determined the glomerular filtration rate. A quantitative assessment of the osmoregulatory function of the kidneys was carried out based on calculations of osmotically purified plasma (Cosm), transport of osmotically free water $(\mathrm{TcH} 2 \mathrm{O})$, and the excreted fraction of osmotically active substances (Efosm). In order to study the state of the hemostasis system, the platelet count and their aggregation activity (ADP inducer $10.0 \mu \mathrm{g} \mathrm{/} \mathrm{ml}$ ), activated partial thromboplastin time (APTT), prothrombin time (PV), thrombin time, relative polymerization time of fibrin monomers (VPFM) were determined, fibrinogen concentration, antithrombin III activity, spontaneous euglobulin lysis time, soluble fibrin - monomer complexes (RFMC) concentration (Technology Standard, Russia). The content of D-dimers was determined on an AS-4 automated analyzer using Helena diagnostic kits (Great Britain). The following were determined: hydroperoxides (diene conjugates and diene ketones) in the blood plasma, the content of malondialdehyde in red blood cells, the activity of superoxide dismutase and the activity of catalase in red blood cells (Solar-300, Belarus). For statistical processing of the obtained results, the software package "STATISTICA 10.0" (StatSoft) and Microsoft Excel 2016 were used. The data are presented as the median (Me) and [25-75] percentiles of the sample. The statistical significance of the differences in the groups of animals was estimated using the nonparametric Mann Whitney $\mathrm{U}$ - test. To assess the statistical dependence of the series of observations, the Spearman rank correlation coefficient $\left(\mathrm{r}_{\mathrm{s}}\right)$ was used. The differences were considered significant with an error probability of $\mathrm{p}<0.05$.

\section{RESULTS AND DISCUSSION}

The experiments showed that the prolonged toxic effect of molybdenum for two months causes a violation of the coagulation system of the blood (Table 1). A decrease in platelet count, and an increase in their aggregation function was observed. The concentration of fibrinogen was higher than the control. Shortening of APTT and elongation of PV were recorded, thrombin time did not change significantly, but the shortening of a more sensitive marker of the state of the final stage of hemocoagulation (VPFM) of fibrin monomer polymerization was revealed. A decrease in AT activity (III) and a decrease in the time of spontaneous euglobulin lysis were recorded. A significant increase in the concentration of RFMC and Ddimers was revealed (Table 1). The data of the experiments demonstrate the development of the state of thrombotic readiness, determined by Momot et al. (2011) [8] as detectable laboratory hypercoagulation with increasing blood levels of hemostasis activation markers, suppression of anticoagulant and fibrinolytic activity. The presented data are confirmed by studies of the toxic effects of nickel, cobalt, lead, and zinc [11].

The hypercoagulable syndrome is characterized by a state of increased readiness of the circulating blood for coagulation [8]. In this case, thrombosis in the vascular bed cannot be detected. However, a number of clinical signs of a prethrombotic state can be observed in the form of an increase in blood viscosity, a slowdown in venous blood flow, transient and initial signs of organ dysfunction, as evidenced by the development of dystrophic and necrobiotic changes in the tissues of the liver, kidneys and heart with toxic effects of molybdenum $[1,12]$.

The experiments showed that the prophylactic administration of melatonin against the background of molybdenum intoxication helps to stabilize the 
hemostasiology picture. The shift in the hemostatic potential towards hypercoagulation was compensated by the activation of anticoagulant and fibrinolytic systems. It can be assumed that melatonin helped to reduce the likelihood of thrombotic readiness, as evidenced by a decrease in the severity of changes in RFMC and D-dimers
(Table 1). The degree of ADP-aggregation increased, the platelet count decreased, but the changes were less pronounced. The fibrinogen content did not statistically differ from that in rats with chronic intoxication, but it did not significantly differ from indicators in intact rats either.

Table 1 Influence of melatonin on indicators of the hemostasis system in rats under chronic molybdene intoxication

\begin{tabular}{|c|c|c|c|}
\hline \multirow{2}{*}{ Experience Conditions } & \multicolumn{3}{|c|}{ Groups of animals } \\
\hline & control & molybdenum & molybdenum and melatonin \\
\hline Platelet count, $10^{9} / 1$ & $534[493-552]$ & $409 * * *[381-449]$ & $470 * / \Delta[437-506]$ \\
\hline ADP-platelet aggregation, $\%$ & $58.3[53.3-63.2]$ & $70.9 * * *[66.7-77.7]$ & $65.1 * / \Delta[62.1-68.2]$ \\
\hline APTT, sec & $26.8[26.0-24.5]$ & $22.7 * * *[22.1-24.1]$ & $25.1 * / \Delta[23.7-26.6]$ \\
\hline Prothrombin time, sec & $17.8[16.6-18.6]$ & $20.56^{* *[19.1-21.7]}$ & $15.8 * / \Delta \Delta \Delta[14.5-17.2]$ \\
\hline Antithrombin (III), \% & $100.7[93.4-112.1]$ & $65.2 * * *[59.3-76.5]$ & $82.8^{* * * / \Delta \Delta[74.6-87.0]}$ \\
\hline Spontaneous euglobulin lysis, min & $510[465-559]$ & $805 * * *[761-869]$ & $421 * / \Delta \Delta \Delta[337-456]$ \\
\hline Fibrinogen, g/l & $2.02[1.65-2.16]$ & $2.69 * * *[2.51-2.9]$ & $2.41[1.95-2.62]$ \\
\hline VPFM, regarding units & $1.03[0.92-1.13]$ & $0.80 * * *[0.68-0.85]$ & $0.87 * / \Delta[0.81-0.97]$ \\
\hline RFMK, mg/100 ml & $2.96[2.53-3.45]$ & $5.31 * * *[4.98-5.83]$ & $4.63 * * * / \Delta[4.2-5.45]$ \\
\hline D-dimer, $\mu \mathrm{g} / \mathrm{L}(\mathrm{DDU})$ & 174 [119-255] & $302 * * *[281-453]$ & $250 \Delta[123-311]$ \\
\hline
\end{tabular}

Note: $*$ / ** / *** $-\mathrm{p} \leq 0.05 / 0.01 / 0.001$ - confidence level regarding intact control,

$\Delta / \Delta \Delta / \Delta \Delta \Delta-\mathrm{p} \leq 0.05 / 0.01 / 0.001-$ relative to the experience with molybdenum.

Several studies demonstrate the association of disorders of vascular-platelet hemostasis with the risk of recurring thrombotic events in patients with chronic kidney disease. The relationship of the intensity of disorders of the components of the hemostatic system with manifestations of renal pathology, developing under the influence of prolonged toxic effects of molybdenum, has not been studied.

An intragastric administration of ammonium molybdate to experimental animals revealed an increase in the volume of 6-hour spontaneous diuresis $(\mathrm{V})$ associated with a decrease in the relative tubular reabsorption of water $\left(\mathrm{RH}_{2} \mathrm{O}\right)$ (Table 2). The level of diuresis was higher due to a significant drop in tubular reabsorption of water ( $p<0.001)$, despite a decrease in glomerular filtration rate (F) (p <0.001). Molybdenum intoxication caused proteinuria and hypoproteinemia. Against the background of increased diuresis, there was an increase in the excreted fraction (Efosm, \%) of osmotically active substances. Due to a simultaneous decrease in the filtration load of nephrons, the osmotic purification (Cosm) of plasma and excretion of osmotically active substances did not significantly change. An increase in urine output and a decrease in osmolarity of urine occurred due to a fall in tubular transport $\left(\mathrm{T}^{\mathrm{C}} \mathrm{H}_{2} \mathrm{O}\right)$ of osmotically free water ( $\mathrm{p}<0.001)$. An analysis of the mechanisms of the revealed abnormalities revealed a negative correlation between the increase in diuresis and the decrease in $\mathrm{T}^{\mathrm{C}} \mathrm{H}_{2} \mathrm{O}$ in the tubules $\left(\mathrm{r}_{\mathrm{s}}=0.67\right)$.

For analyzing the mechanisms of reducing tubular reabsorption of water in rats during chronic intoxication, we studied the urea-sodium profile of the layers of kidney tissue (Table 3). In experimental animals with intragastric administration of a molybdenum salt under conditions of spontaneous diuresis, the concentration gradient of sodium and urea in the layers of the kidney tissue from the cortex to the papilla decreased relative to the control indices. Therefore, a decrease in the concentration transport of osmotically free water and the diuretic effect of ammonium molybdate can be associated with a decrease in the cortico-papillary osmotic gradient in animals with molybdenum intoxication.

An analysis of the results of the experiment revealed correlations between the dynamics of hemostasiological parameters and the state of the urinary function of the kidneys in rats with chronic intoxication. A decrease in 
tubular reabsorption of water correlated with an increase in the degree of thrombinemia $\left(r_{s}=-0.67 ; p<0.05\right)$. A high degree of inverse correlation of proteinuria level with a decrease in antithrombin activity was revealed $\left(r_{\mathrm{s}}=-0.78\right.$; $\mathrm{p}<0.05)$.

Table 2 Influence of melatonin on the basic processes of urine formation and excretion of protein with urine in rats under chronic molibbene intoxication

\begin{tabular}{|c|c|c|c|}
\hline \multirow{2}{*}{ Experience Conditions } & \multicolumn{3}{|c|}{ Groups of animals } \\
\hline & control & molybdenum & molybdenum and melatonin \\
\hline Diuresis, V, (ml/h/100g) & $0.07[0,067-0,056]$ & $0.135 * * *[0,127-0,137]$ & $0.123 * * * / \Delta \Delta \Delta[0.114-0.127]$ \\
\hline Glomerular filtration of water, $\mathrm{F}(\mathrm{ml} / \mathrm{h} / 100 \mathrm{~g})$ & $20.6[18.6-21.3]$ & $14.35 * * *[13.36-14.5]$ & $16.8 * * * / \Delta[15.0-17.34]$ \\
\hline $\begin{array}{l}\text { Relative tubular reabsorption of water } \mathrm{R}_{\mathrm{H} 2 \mathrm{O}} \\
(\%)\end{array}$ & $99.65[99.61-99.69]$ & 99.03***[98.99-99.08] & $99.26 * * * / \Delta \Delta \Delta[99.18-99.3]$ \\
\hline Osmolarity of urine, Uosm (mosm / ml) & $1.594[1.53-1.619]$ & $0.796 * * *[0.739-0.842]$ & $0.983 * * * / \Delta \Delta[0.909-1.019]$ \\
\hline $\begin{array}{l}\text { Osmotic Excretion of osmotically active } \\
\text { substances, Uosm x V (mosm/h/100 g) }\end{array}$ & $0.112[0.102-0.123]$ & $0.108[0.102-0.111]$ & $0.118[0.106-0.122]$ \\
\hline Osmotic cleansing Cosm $(\mathrm{ml} / \mathrm{h} / 100 \mathrm{~g})$ & $0.381[0.337-0.406]$ & $0.362[0.336-0.369]$ & $0.393[0.354-0.413]$ \\
\hline $\begin{array}{l}\text { Excreted fraction of osmotically active } \\
\text { substances Efosm }(\%)\end{array}$ & $1.893[1.636-2.153]$ & $2.566 * * *[2.44-2.736]$ & $2.259 * / \Delta[2.138-2.461]$ \\
\hline $\begin{array}{l}\text { Osmotically free water transport, } \mathrm{T}^{\mathrm{C}} \mathrm{H}_{2} \mathrm{O} \\
(\mathrm{ml} / \mathrm{h} / 100 \mathrm{~g})\end{array}$ & $0.311[0.27-0.331]$ & $0.223 * * *[0.199-0.243]$ & $0.277 * / \Delta \Delta \Delta[0.239-0.289]$ \\
\hline Proteinuria Uosm (mg/ml) & $0.083[0.078-0.097]$ & $0.968 * * *[0.884-1.066]$ & $0.756^{* * *} / \Delta \Delta[0.659-0.848]$ \\
\hline
\end{tabular}

Note: see Table 1.

Table 3 Influence of melatonin on the urea and sodium content in the layers of the kidney tissue under conditions of spontaneous diuresis in rats under chronic molibbene intoxication

\begin{tabular}{|c|c|c|c|}
\hline \multirow{2}{*}{$\begin{array}{l}\text { Experience } \\
\text { Conditions }\end{array}$} & \multicolumn{3}{|c|}{ Groups of animals } \\
\hline & control & molybdenum & molybdenum and melatonin \\
\hline \multicolumn{4}{|c|}{ Urea in the layers of the kidney tissue ( $\mathrm{mmol} / 100 \mathrm{~g}$ wet weight) } \\
\hline Bark & $19.93[18.56-20.6]$ & $18.7[17.61-19.28]$ & $19.55[18.49-2017]$ \\
\hline Medulla & $57.82[56.21-58.9]$ & $48.45[46.92-49.0]^{* * *}$ & $50.04[48.49-51.20] * * * / \Delta$ \\
\hline Papilla & $167.4[162.1-171.8]$ & $134.0[125.8-135.9] * * *$ & $145.7[139.0-152.7] * * * / \Delta \Delta \Delta$ \\
\hline \multicolumn{4}{|c|}{ Sodium in the layers of the kidney tissue ( $\mathrm{mmol} / \mathrm{kg}$ dry weight) } \\
\hline Bark & $24,2[23,51-24,68]$ & $23,21[21,74-24,1]$ & $24,5[23,3-25,1]$ \\
\hline Medulla & $39,9[39,2-41,9]$ & $37,8[36,9-39,9]$ & $38,7[37,5-39,9]$ \\
\hline Papilla & $98,33[94,4-101,1]$ & $83,02[80,49-88,62] * * *$ & $91,06[87,16-95,23] * * / \Delta \Delta$ \\
\hline
\end{tabular}

Note: see Table 1. 
Isolated administration of melatonin at a dose of $5 \mathrm{mg} / \mathrm{kg}$ of weight in the control group of rats did not cause significant changes in the main processes of urination. However, there was a tendency to increase the volume of spontaneous 6-hour diuresis.

In the combined model with the simultaneous administration of the molybdenum salt and melatonin, polyuria was higher than in intact rats. However, its level was lower than in the group of animals treated only with metal. In this case, an increase in diuresis was also due to inhibition of tubular reabsorption of water $(\mathrm{p}<0.001)$, but less pronounced than in molybdenum rats $(\mathrm{p}<0.001)$. The level of proteinuria was lower than with the isolated administration of metal ( $p<0.01)$.

The experiments revealed a decrease in urine osmolality ( $p<0.001)$ against a background of a less pronounced decrease in the cortico-papillary gradient of the kidneys and tubular transport of osmotically free $\mathrm{T}^{\mathrm{C}} \mathrm{H}_{2} \mathrm{O}$ water $(\mathrm{p}<0.001)$ under conditions of spontaneous diuresis, which may indicate the restoration of the concentration function of the kidneys.

Correlation analysis established the parameters of the urinary system and indicators of the coagulation system in rats against the background of prophylactic administration of melaxen. This analysis revealed a reliable statistical relationship between the decrease in the degree of proteinuria and the restoration of ATIII activity $\left(r_{s}=-\right.$

Table 4 Influence of melatonin on ratal indicators in rats under chronic molibbene intoxication

\begin{tabular}{|c|c|c|c|}
\hline \multirow{2}{*}{ Experience Conditions } & \multicolumn{3}{|c|}{ Groups of animals } \\
\hline & control & molybdenum & molybdenum and melatonin \\
\hline Malondialdehyde $(\mu \mathrm{mol} / \mathrm{L})$ & $29.89[27.79-30.92]$ & $39.45 * * *[37.28-41.88]$ & $32.82 * * / \Delta \Delta \Delta[30.95-35.55]$ \\
\hline The content of hydroperoxides (233 nm) & $0.062[0.058-0.070]$ & $0.085 * * *[0.079-0.093]$ & $0.073 * / \Delta \Delta[0.067-0.078]$ \\
\hline The content of hydroperoxides (278 nm) & $0.0459[0.040-0.048]$ & $0.021 * * *[0.016-0.026]$ & $0.031 * * / \Delta \Delta[0.025-0.037]$ \\
\hline $\begin{array}{l}\text { The activity of superoxide dismutase (unit } \\
\text { Siberian., \%) }\end{array}$ & $70.34[64.27-73.36]$ & $57.11 * *[49.8-61.93]$ & $63.06 * / \Delta[60.23-67.24]$ \\
\hline Catalase activity $(* 10-4 \mathrm{ME} / \mathrm{gHb})$ & $7.63[6.82-8.55]$ & $5.53 * * *[4.28-6.04]$ & $6.87 * / \Delta[5.85-7.2]$ \\
\hline
\end{tabular}

Note: see Table 1.

Intragastric administration of the antioxidant melaxen caused an increase in the level of catalase activity in erythrocytes ( $p<0.05)$, a decrease in the concentration of malondialdehyde $(\mathrm{p}<0.001)$ and a restoration of the level of hydroperoxides ( $p<0.01$ and $p<0.01)$ in the blood plasma. When analyzing the obtained experimental data, correlations were established between the positive dynamics of hemostasis system indicators, urinary function of the kidneys, and restoration of activity of LPO processes. A significant positive correlation was found between the restoration of catalase activity and an increase in antithrombin III activity $\left(\mathrm{r}_{\mathrm{s}}=0.64 ; \mathrm{P}<0.05\right)$, as well as a high degree of inverse correlation with the time of spontaneous euglobulin lysis $\left(\mathrm{r}_{\mathrm{s}}=0.57 ; \mathrm{P}<0.05\right)$. The
$0.55 ; \mathrm{p}<0.05)$ and the time of spontaneous euglobulin lysis $\left(\mathrm{r}_{\mathrm{s}}=0.68 ; \mathrm{p}<0.05\right)$. A decrease in the level of thrombinemia was closely associated with the restoration of tubular reabsorption of water $\left(r_{s}=-0.64 ; p<0.05\right)$ under the influence of melatonin.

It was previously shown that oxidative stress is one of the main pathogenetic mechanisms of the nephrotoxic effect of heavy metal compounds. At the same time, mechanisms of the relationship between the activity of hemocoagulation and lipoperoxidation systems in many pathological conditions, including renal pathology, are described. An increase in the content of the following indicators was revealed: lipid peroxidation products in blood plasma, erythrocytes, leukocytes and platelets during hyperoxidation [6]. In this regard, it is justified to use drugs that have an inhibitory effect on LPO processes to prevent experimental chronic molybdenum coagulopathy and nephropathy $[2,3]$.

The results of the experiments demonstrate an increase in the activity of LPO processes and inhibition of the antioxidant enzyme link with the development of oxidative stress (Table 4). There was an increase in the concentration of malondialdehyde $(\mathrm{p}<0.001)$, an increase in the level of hydroperoxides in the blood plasma ( $p$ $<0.001$ and $p<0.001)$ and a decrease in the activity of SOD $(\mathrm{p}<0.01)$ and catalase $(\mathrm{p}<0.001)$. 


\section{CONCLUSION}

The prophylactic use of melatonin reduces the severity of changes in the indicators of vascular-platelet and coagulation hemostasis. It also restores the activity of the anticoagulant and fibrinolytic blood systems and the level of thrombinemia. Thus, this prevention contributes to the restoration of hemostatic balance.

Intragastric administration of melatonin softens the severity of the nephrotoxic effects of ammonium molybdate. Also, intragastric administration of melatonin has a beneficial effect on the preservation of indicators of the functional state and concentration ability of the kidneys.

The prophylactic administration of melatonin prevents the decrease in the activity of the antioxidant system and promotes the utilization of lipid peroxidation products. Thus, the prophylactic administration of melatonin eliminates the imbalance in the lipid peroxidation system and antioxidant protection.

The results of the study allow recommending the use of melatonin to develop methods for correcting hemostasis disorders when molybdenum compounds are exposed to the body.

\section{REFERENCES}

[1] A.P. Avtsin, Human microelementoses: etiology, classification, organopathology, Medicine, Moscow, 1991, $496 \mathrm{p}$.

[2] E.B. Arushanyan, The effect of melatonin on platelet hemostasis and its circadian organization, Expert. and wedge. Pharmacol. 76(5) (2013) 32-38.

[3] E.B. Arushanyan, K.B. Ovanesov, The significance of melatonin for kidney activity, Med. Bull. of the North Caucasus 1(1) (2018) 120-125.

[4] V.B. Breen, E.R. Melikova, J.K. Albegova, E.M. Gagloeva, Changes in the urinary function of the kidneys under the influence of ammonium molybdate in intact rats and under experimental hypercalcemia, Nephrol. 18(5) (2014) 63-69.

[5] V.B. Brin, K.G. Mittsiev, O.T. Kabisov et al., Experimental therapy of changes in blood circulation and electrolyte content during cadmium intoxication, Med. Bull. of the North Caucasus 13(4) (2018) 246.

[6] A.Sh. Byshevsky, S.L. Galyan, I.V. Ralchenko, The effect of a combination of antioxidant vitamins on hemostasis in experimental hyperoxidation, Experim. and Clin. Pharmacol. 68(3) (2005) 34-36.

[7] N.V. Reutova, Analysis of the incidence of the population in the area of the tungsten-molybdenum plant, Hygiene and sanitat. 4 (2007) 13-15.

[8] A.P. Momot, L.P. Tsyvkina, I.A. Taranenko, Modern methods of recognition of thrombotic readiness, Alt. Univ., Barnaul, 2011, 138 p.

[9] M. Anke, S. Holzinger, M. Seifert et al., The biological and toxicological importance of molybdenum in the environment and in the nutrition of plants, animals and man, Acta Alimentaria 39(1) (Mar. 2010) 1-11.

[10] C.A. Goodwin, C.P. Wheeler-Jones, S. Namiranian, Increased expression of procoagulant activity on the surface of human platelets exposed to heavy-metal compounds, Biochem. J. 15(308) (1995) 1-21.

[11] N.J. Mutch, E.K. Waters, J.H. Morrissey, Immobilized transition metal ions stimulate contact activation and drive factor XII-mediated coagulation, J. Thromb. Haemost. 10(10) (2012) 2108-2115.

[12] R. Pandey, R. Kumar, S. Singh, S. Srivastava, Molybdenum in rat tissue, Experim. Toxicol. 21 (2002) 33-35.

[13] A. Romero, E. Ramos, Los Ríos et al., A review of metal-catalyzed molecular damage: protection by melatonin, J. of Pineal Res. 56(4) (2014) 343-370. 